\title{
PERDAS EXTREMAS EM MERCADOS DE RISCO
}

\author{
EXTREME LOSSES IN RISK MARKETS
}

\author{
RONALDO A. ARRAES \\ Professor Adjunto, PhD, do Programa de Pós-Graduação em Economia - CAEN \\ da Universidade Federal do Ceará - CE \\ E-mail: ronald@ufc.br \\ ALANE S. ROCHA \\ Professora Ms. do Departamento de Administração \\ da Faculdade de Economia, Administração, Atuária e Contabilidade \\ da Universidade Federal do Ceará - CE \\ E-mail: alanesiqueira@terra.com.br
}

\section{RESUMO}

Neste artigo, infere-se sobre a distribuição de valores extremos de uma variável aleatória representada pelas severas perdas diárias em investimentos financeiros. A Teoria dos Valores Extremos (TVE) fundamenta a modelagem de eventos gravosos raros, com expressivas conseqüências econômicas associadas a probabilidades muito pequenas de ocorrerem. Uma das grandes preocupações, na análise de riscos, é desenvolver técnicas para prever essas ocorrências excepcionais. Assim, as caudas das distribuições desses eventos raros são importantes para o estudo do risco, tornando a TVE uma ferramenta de grande valia para a estimação mais acurada do risco dessas perdas elevadas. Investigou-se, neste trabalho, a estimação de perdas máximas esperadas para séries financeiras, empregando-se: i) métodos tradicionais, que utilizaram todos os dados amostrais para analisar a variável aleatória em questão e.ii) a metodologia dos Valores Extremos, particularmente a da Distribuição Generalizada dos Valores Extremos (DGVE), que utilizou apenas um conjunto de máximos amostrais para a estimação das perdas máximas esperadas. Concluiu-se que os métodos tradicionais subestimaram as perdas esperadas, sobretudo nas proximidades dos limites das caudas das distribuições, e que a DGVE mostrou-se bem mais eficiente na previsão dessas perdas extremas nas séries analisadas: Ibovespa, Merval, Dow Jones.

Palavras-chave: Risco Financeiro; Distribuição dos Valores Extremos (TVE); Valor em Risco (VaR); Perdas Extremas.

\section{ABSTRACT}

This paper aims to infer about the distribution of extremes values of a continuous random variable, represented as the severe daily losses in financial markets investments. The Extreme Value Theory (EVT) plays a fundamental role in modeling rare events associated with great losses and very small probabilities of occurrence. One of the great concerns in risk management is to develop analytic techniques to foresee those exceptions. In that way, the tails of the rare losses' probability density function ( $p d f$ ) are of great importance in evaluating that kind of risk, turning EVT into a valuable tool for an accurate evaluation of high loss risks. The estimations of expected maximum losses in financial series are investigated by means of: i) traditional methods, which used all sample data in fitting the random variable pdf; ii) the Extreme Value methodology, particularly the Generalized Extreme Value distribution (GEV), which only used a set of maximum values detected in the sample data in estimating the pdf of expected maximum losses. The findings indicate, firstly, an important underestimation of extreme losses with the traditional methods, mainly in the pdf lower tail limits, and, secondly, that the GEV distribution proved to be more efficient in forecasting extreme losses in the analyzed series: Ibovespa, Merval, Dow Jones.

Keywords: Financial Investment Risk; Distribution of Extreme Values; Value-at-Risk (VaR); Extreme Losses. 


\section{INTRODUCÃO}

O homem sempre buscou segurança e proteção contra acontecimentos indesejáveis. Em tempos pré-históricos, as pessoas preocupavam-se com sua integridade física em decorrência da agressão das forças brutas da natureza. Com o progresso da civilização, a questão da segurança tem se tornado mais complexa, envolvendo numerosos riscos novos e incorporando sofisticadas técnicas de estruturação de proteções a esses riscos.

Há dois tipos principais de riscos econômicos: 1) o risco dos investidores, que envolve a chance de perda ou de ganho e o 2) o risco puro, quando há, apenas, a chance de perda. Os negócios de seguro são do tipo riscos econômicos puros, porquanto perder a vida, ou o carro ou o apartamento não tem a contrapartida de nenhum ganho.

A Teoria dos Valores Extremos - TVE desempenha papel fundamental na modelagem de eventos associados a probabilidades muito pequenas ou eventos raros para a quantificação, dentre outras, de grandes riscos econômicos. Vários são os domínios de aplicação da TVE, tendo, como exemplo, as companhias resseguradoras ${ }^{1}$ que necessitam prever, adequadamente, os montantes de grandes indenizações com seguros. O comentário abaixo evidencia a necessidade do aprimoramento na quantificação de riscos econômicos.

For the first time in the history of our planet, mankind is about to change the climate significantly and possibly irreversibly, without having any idea of the consequences that will have[....] With economic and insured losses increasing in volume by a factor of 3 and 5 respectively since the 1960s, we definitely have a trend which, without exaggeration, may be regarded as dramatic. (Munich Re, 1990)²

A ocorrência de eventos com valores elevados implica certos custos para remediar suas conseqüências. O terremoto de Kobe, por exemplo, acarretou pagamento de indenizações entre $\$ 100$ e $\$ 150$ bilhões de dólares pelas companhias de seguro.

Souza (1999, p. 41) evidencia a importância da TVE no estudo e dimensionamento de infra-estrutura de apoio a catástrofes naturais:

[...] Um caso bastante citado é o dimensionamento dos diques construídos nos Países Baixos para controlar o avanço do mar. O último grande transbordamento registrado teve conseqüências desastrosas, matando mais de 1800 pessoas em 1953. O trabalho de matemáticos holandeses como Van Dantzig foi decisivo para o avanço tanto da TVE como para o dimensionamento da altura dos diques. O problema nesse caso é: dadas as observações do nível máximo anual observado (o maior deles antes de 1953, quando o nível atingiu $3.85 \mathrm{~m}$ acima do normal, tinha sido na enchente de
1570, de $4 \mathrm{~m}$ ) qual a maior altura que o nível do mar atingirá nos próximos 10.000 anos (quantil associado à probabilidade de 1-10-4)? Van Dantzig estimou como sendo $5.14 \mathrm{~m}$ acima do nível de referência. Trata-se de um exemplo típico onde é necessário se fazer inferência para eventos fora do conjunto amostral.

A aplicação de recursos e o estabelecimento de metas de desempenho são atividades constantes dos fundos de pensão. Para o cumprimento de tais metas, torna-se necessário obter retornos em um ambiente sob condições de risco. Com isso, a adequada identificação, mensuração e controle dos riscos é de vital importância. Os riscos do ativo englobam todas as razões que fazem os rendimentos obtidos nos diversos portfolios de investimentos do plano se distanciarem dos retornos intencionados, comprometendo, se a menor, o processo de fundação de suas obrigações previdenciais. Para os fundos de pensão, o domínio das melhores técnicas de gestão de riscos, com o intuito de proteção de seu patrimônio, revela-se essencial na tarefa de maximizar a probabilidade de alcançar o desejado equilíbrio econômico-financeiro de um plano de aposentadoria. A aplicação da TVE torna-se, então, relevante para uma previsão mais acurada dos riscos econômicos de grandes proporções que podem influenciar negativamente os retornos das aplicações dos fundos de pensão.

O desenvolvimento do mercado financeiro intensificou-se na década de 90 impulsionando a implementação de instrumentos de acompanhamento gerenciais voltados à nova realidade da globalização. As conseqüências de eventos específicos, como a crise da Rússia, são difundidas rapidamente no mercado financeiro mundial.

As instituições financeiras mantêm modelo interno de análise de risco de mercado, com destaque especial para o RiskMetrics, desenvolvido pelo banco americano J.P. Morgan, que teve como resultado final a técnica de mensuração de riscos conhecida como Value-at-Risk (VaR).

Morgan (1996, p. 5) refere-se à medida Valor em Risco (VaR) como sinônimo de risco de mercado:

Over the last few years measures of market risk have

become synonymous with the term Value-at-Risk.

Jorion (1997, p. 18) apresenta o seguinte conceito para o VaR:

O VaR sintetiza a maior (ou pior) perda esperada dentro de determinado período de tempo e intervalo de confiança.

Em 1993, o Grupo dos Trinta (G-30), uma equipe composta por banqueiros, agentes financeiros e acadêmicos

1 O resseguro pode ser resumidamente definido como o seguro do seguro. É um instrumento de transferência de risco das seguradoras para as resseguradoras com a finalidade de evitar o comprometimento de sua saúde financeira.

2 CHAVEZ, V.; ROEHRL, A. Extreme Value Theory can save your neck, 2004. Disponível em <http://www.approximity.com/papers/TVE wp.pdf>. Acesso em: 25 de janeiro de 2005 
importantes das maiores nações industriais, emitiu relatório contendo uma recomendação importante: a avaliação de posições no mercado e mensuração de riscos financeiros mediante metodologia de Value-at-Risk (VaR).

Sua capacidade de sintetizar, em um único número, a perda financeira esperada com determinado grau de confiança, foi decisiva para sua recomendação pelo Comitê da Basiléia como metodologia para a alocação de capital em instituições financeiras.

A Teoria dos Valores Extremos contribuiu para o aprimoramento dos modelos de VaR por concentrar-se no ajuste da distribuição apenas sobre os valores extremos da variável aleatória, diminuindo, portanto, a influência dos valores centrais. A TVE auxilia, portanto, na estimação das perdas máximas esperadas em momentos de instabilidade. Em suma, o resultado fundamental da TVE consiste na convergência da distribuição assintótica de uma série de mínimos (máximos) padronizados.

Objetiva-se, então, neste trabalho: i) aplicar a TVE a amostras de dados financeiros e ii) comparar as estimativas de perdas máximas encontradas a partir da TVE com as estimativas de perdas máximas encontradas pelas metodologias tradicionais, as quais, diferentemente da TVE, estimam perdas esperadas utilizando a amostra total de dados.

Seguindo-se a essa introdução, a seção 1 traz uma abordagem teórica geral sobre a TVE. Na seção 2, são enfocados os aspectos metodológicos e as séries utilizadas para a estimação das perdas esperadas. A seção 3 trata das metodologias de estimação de perdas máximas, comparando-se as estimativas de perdas máximas esperadas com as perdas efetivamente ocorridas. A última seção é reservada às conclusões.

\section{BASE TEÓRICA}

O objetivo deste trabalho é fazer inferências sobre os extremos de uma variável aleatória, sejam extremos pertencentes à cauda inferior da variável, como mínimos de retornos diários de ativos financeiros, ou extremos pertencentes a sua cauda superior, como o valor de uma indenização de sinistro. Assim, o propósito do administrador de risco é, em última instância, constituir reservas suficientes para se resguardar contra esses eventos extremos.

O resultado fundamental da Teoria dos Valores Extremos consiste na convergência da distribuição assintótica de uma série de mínimos ou máximos padronizados para distribuições pertencentes às seguintes classes de distribuições: i) Distribuição Generalizada do Valor Extremo - DGVE ou ii) Distribuição Generalizada de Pareto - DGP.

A Teoria de Valores Extremos, portanto, concentra-se em classes especiais de distribuições de probabilidade: as distribuições do tipo DGVE, classe que inclui as distribuições de Gumbel, de Fréchet e de Weibull, e as distribui- ções DGP, como a Exponencial, a Pareto e a Beta. Na forma padronizada, a DGVE e a DGP dependem apenas de um parâmetro, chamado de índice de cauda.

Para as distribuições DGVE e DGP utilizam-se, respectivamente, as seguintes formas de tratar os valores extremos: a primeira abordagem considera máximos (ou mínimos) selecionados em períodos sucessivos, por exemplo, meses ou anos. Essas observações selecionadas constituem os eventos extremos. Na figura $1 \boldsymbol{0}$, as observações $X_{2}, X_{5}$, $X_{7}$ e $X_{11}$ representam os máximos para quatro blocos com períodos de três observações. A segunda abordagem seleciona extremos que excedem um determinado limite. As observações $X_{1}, X_{2}, X_{7}, X_{8}, X_{9}$ e $X_{11}$ na figura $2 \mathbf{0}$, excedem o limite $\boldsymbol{u}$ e constituem eventos extremos.

A primeira técnica apresenta a desvantagem da possibilidade, dependendo do tamanho do bloco, de perder observações extremas dentro do mesmo bloco. Observa-se, na figura 1, que no terceiro bloco desconsidera-se um valor

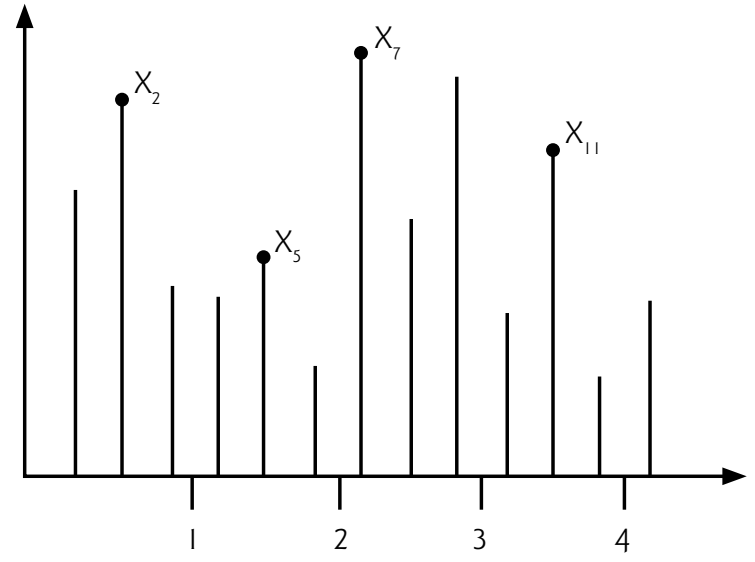

Figura 1

Blocos de Máximo

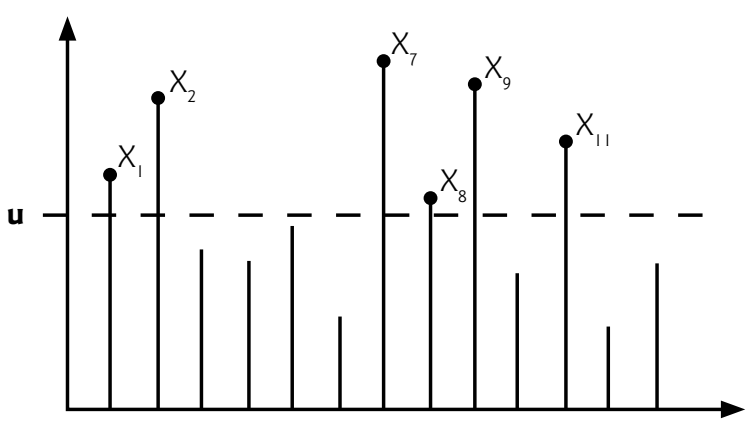

Figura 2

Excesso ao limite $u$ 
maior que os máximos $x_{2}, x_{5}$ e $x_{11}$. A escolha do tamanho do bloco pode, entretanto, ser realizada de forma objetiva como, por exemplo, blocos de meses, blocos de anos.

Na segunda técnica, a escolha de um limite suficientemente alto é menos objetiva. A escolha está sujeita a um trade-off entre variância e viés. Aumentando o número de observações para as séries de máximos (baixo limite) são introduzidas nas séries algumas observações do centro da distribuição, tornando a estimativa do parâmetro índice de cauda mais consistente, porém mais viesada. Por outro lado, escolhendo um limite alto reduz-se o viés do estimador, tornando-o, entretanto, mais impreciso, em virtude da utilização de uma menor quantidade de observações para a sua estimação.

Os comentários, a partir desse ponto, referem-se ao máximo, pois os resultados para o mínimo podem ser obtidos pela relação,

$$
\operatorname{minimo}\left(X_{1}, \ldots, X_{n}\right)=-\operatorname{máximo}\left(-X_{1}, \ldots,-X_{n}\right) \text {. }
$$

Assim, tratou-se aqui a TVE aplicando apenas a DGVE.

\subsection{Distribuição Generalizada dos Valores Extremos - DGVE}

A Distribuição Normal é uma importante distribuição limite para soma de médias amostrais, como explicitado no Teorema do Limite Central. Similarmente, a família de distribuições de valores extremos representa as distribuições limite para os máximos amostrais, como determinado pelo Teorema de Fisher $\varepsilon$ Tippett, tal como exposto abaixo. Essa família de distribuições de valores extremos determina a forma da distribuição limite (assintótica) do máximo à medida que a extensão do intervalo de tempo, a partir do qual o máximo é selecionado, tende para infinito. A função repartição Fx dos máximos DGVE, admitindo a hipótese de independência ${ }^{3}$ para os máximos, é denotada, em sua forma não padronizada, por: (MENDES, 2004, p. 45).

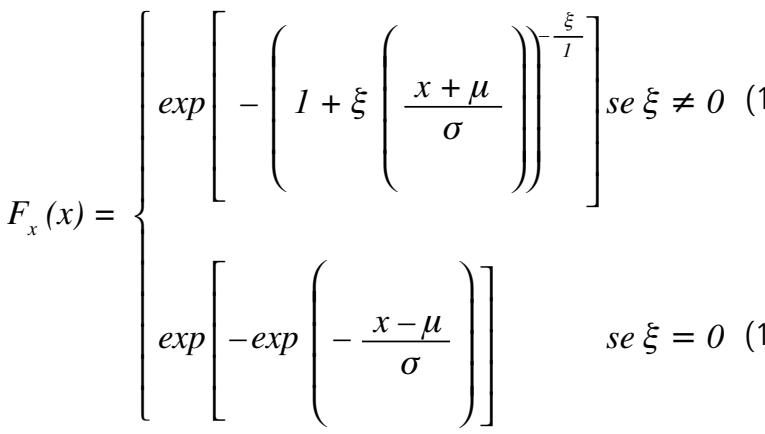

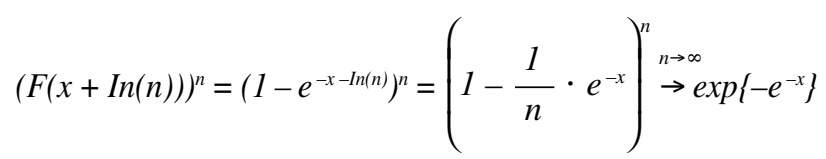

Vê-se que tomando como constantes normalizadoras $c_{n}=1$ e $d_{n}=\ln (n)$, a distribuição assintótica do máximo coletado em bloco de tamanho $n$ de observações oriundas de uma exponencial unitária será, quando $\mathrm{n} \rightarrow \infty$ uma distribuição de Gumbel.

\footnotetext{
3 Essa hipótese é bastante plausível, pois, eventos raros em geral são determinados por circunstâncias muito particulares, e que dificilmente se reproduziriam no futuro. São imprevisíveis e típicos de momentos de crises.

4 Para mais detalhes sobre a Teoria dos Valores Extremos ver: MENDES, Beatriz V. de M. Uma Introdução à Analise de Valores Extremos. Rio de Janeiro: E-papers Serviços Editoriais, 2004

5 Uma distribuição de probabilidade é degenerada quando sua média e variância são iguais a zero.
} 
A função densidade de probabilidade da DGVE, para $\xi \neq 0$, é encontrada derivando-se a equação (1.1) acima e para $\xi=0$ derivando-se a equação (1.2), logo:

$$
f_{x}(x)= \begin{cases}\frac{1}{\sigma}\left[1+\xi\left(\frac{x-\mu}{\sigma}\right)\right]-\left(\frac{1+\xi}{\xi}\right) \exp \left\{-\left[1+\xi\left(\frac{x-\mu}{\sigma}\right)\right]^{-\frac{\xi}{1}}\right\} \text { se } \xi \neq 0 \\ \frac{1}{\sigma}\left\{\exp \left(-\frac{x-\mu}{\sigma}\right) \exp \left[-\exp \left(-\frac{x-\mu}{\sigma}\right)\right]\right\} & \text { se } \xi=0\end{cases}
$$

definidas em, $-\infty<\mathrm{x}<\mu-\sigma / \xi$ para $\xi<0 ; \mu-\sigma / \xi<\mathrm{x}<$ $+\infty$ para $\xi>0$ e $-\infty<x<+\infty$ para $\xi \rightarrow 0$.

Os parâmetros $\mu$ e $\sigma$ correspondem, respectivamente, a parâmetros de escala e de tendência; o terceiro parâmetro, $\xi$, também representado por $\alpha=1 / \xi$, indica a densidade da cauda. O índice da cauda, $\alpha$, mede a velocidade com que a cauda da distribuição se aproxima de zero.

O parâmetro $\alpha$, designado de índice da cauda, modela a cauda da distribuição. De acordo com o valor do índice da cauda, três tipos de distribuições do valor extremo são obtidos: a distribuição Fréchet $(\alpha>0)$, a distribuição de Gumbel $(\alpha=0)$ e a distribuição de Weibull $(\alpha<0)$. Esse resultado é muito significativo, pois como a distribuição assintótica do máximo sempre pertence a uma dessas três distribuições, qualquer que seja a distribuição original, nenhuma suposição é necessária sobre a natureza da distribuição original das observações.

As caudas das DGVE podem ser representadas por distribuições contínuas, em que a cauda da distribuição de Gumbel corresponde às distribuições exponencial, gama, normal ou log-normal, a da distribuição de Fréchet segue uma distribuição de Cauchy, Pareto ou t-Student e a da distribuição de Weibull segue uma distribuição Uniforme. (GNEDENKO ${ }^{6}$ (1943) apud BAUTISTA (2002, p. 3)).

\subsection{Estimação da DGVE}

Para a estimação dos parâmetros $\xi, \mu, \sigma$ da DGVE, utilizar-se-á o método da máxima verossimilhança.

As estimativas dos parâmetros são, para a DGVE, encontradas utilizando-se a função de log-verossimilhança dada por:

$$
l(\xi, \mu, \sigma ; x)=\operatorname{In}(L(\xi, \mu, \sigma ; x))=\operatorname{In}\left(\prod_{i} f\left(x_{i}\right)\right)
$$

em que: $f\left(x_{i}\right)$ é dada pela equação (1.3) ou (1.4).

Para as distribuições de Fréchet e Weibull, representadas pela equação (1.3), desenvolve-se a função log-verossimilhança.

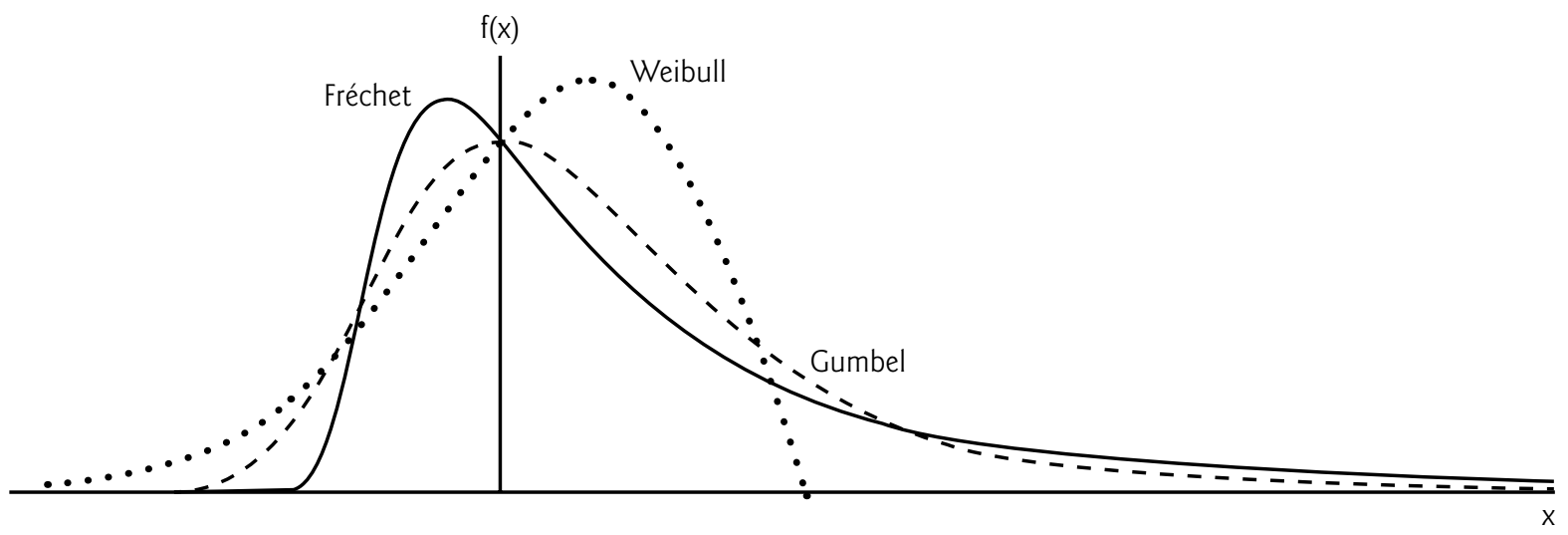

Figura 3

Função densidade de probabilidade GVE para $\xi=-0,5$ (Weibull), $\xi \rightarrow 0$ (Gumbel) e $\xi=0,5$ (Fréchet) com $\mu=0$ e $\sigma=1$ 


$$
\begin{aligned}
l(\xi, \mu, \sigma ; x) & =\operatorname{In}\left(\prod_{i} f\left(x_{i}\right)\right) \\
& =\operatorname{In}\left\{\prod_{i=1}^{n}\left\{\frac{1}{\sigma}\left[1+\xi\left(\frac{x-\mu}{\sigma}\right)\right]-\left(\frac{1+\xi}{\xi}\right) \exp \left\{-\left[1+\xi\left(\frac{x-\mu}{\sigma}\right)\right]^{-\frac{1}{\xi}}\right]\right\}\right\} \\
& =-n \ln \sigma-\left(\frac{1+\xi}{\xi}\right) \sum_{i=1}^{n} \operatorname{In}\left[1+\xi\left(\frac{x_{i}-\mu}{\sigma}\right)\right]-\sum_{i=1}^{n}\left[1+\xi\left(\frac{x_{i}-\mu}{\sigma}\right)\right]^{-\frac{1}{\xi}} \\
& =\sum_{i=1}^{n}\left\{-\ln \sigma-\left(\frac{1+\xi}{\xi}\right) \cdot \operatorname{In}\left[1+\xi\left(\frac{x_{i}-\mu}{\sigma}\right)\right]-\left[1+\xi\left(\frac{x_{i}-\mu}{\sigma}\right)\right]^{-\frac{1}{\xi}}\right\}
\end{aligned}
$$

Os estimadores de máxima verossimilhança são, então, obtidos pela solução do sistema de equações não lineares formado pelas derivadas de primeira ordem da equação
(1.6), em relação a cada parâmetro $\xi, \mu, \sigma$, derivadas estas igualadas a zero. Referido sistema de equações está apresentado abaixo.

$$
\begin{aligned}
& \frac{1}{\hat{\sigma}} \sum_{i=1}^{n}\left(\frac{1+\hat{\xi}-w_{i}^{-\frac{1}{\hat{\xi}}}}{\hat{\sigma}}\right)=0 \\
& -\frac{n}{\hat{\sigma}}+\frac{1}{\hat{\sigma}^{2}} \sum_{i=1}^{n}\left\{\frac{\left(x_{i}-\hat{\mu}\right)\left[(1+\hat{\xi})-w_{i}-\frac{1}{\hat{\xi}}\right]}{w_{i}}\right\}=0 \\
& \sum_{i=1}^{n}\left\{\left(1-w_{i}^{-} \frac{1}{\hat{\xi}}\right)\left[\frac{1}{\hat{\xi} 2} \operatorname{In} w_{i}-\frac{\left(x_{i}-\hat{\mu}\right)}{\hat{\xi} \cdot \hat{\sigma} \cdot w_{i}}\right]-\frac{\left(x_{i}-\hat{\mu}\right)}{\hat{\sigma} \cdot w_{i}}\right\}=0 \\
& \text { em que: } w_{i}=1+\hat{\xi}\left(\frac{x_{i}-\hat{\mu}}{\sigma}\right)
\end{aligned}
$$

Como o sistema de equações acima não possui solução analítica, métodos numéricos são utilizados.

Para a distribuição de Gumbel, representada pela equação
(1.4), tem-se a seguir: i) a função log-verossimilhança e ii) o sistema de equações para estimação dos parâmetros $\mu \mathrm{e}$.

i) função log-verossimilhança:

$$
\begin{aligned}
l(\xi, \mu, \sigma ; x) & =\operatorname{In}\left(\prod_{i} f\left(x_{i}\right)\right) \\
& \left.=\operatorname{In}\left\{\prod_{i=1}^{n}\left\{\frac{1}{\sigma} \cdot\left\{\exp \left(-\frac{x-\mu}{\sigma}\right) \exp \left[-\exp \left(-\frac{x-\mu}{\sigma}\right)\right]\right\}\right\}\right\}\right] \\
& =\sum_{i=1}^{n}\left\{-\ln \sigma-\left(\frac{x_{i}-\mu}{\sigma}\right)-\exp \left(-\frac{x_{i}-\mu}{\sigma}\right)\right\}
\end{aligned}
$$


ii) sistema de equações não-lineares para estimação dos parâmetros da DGVE - Gumbel:

$-\frac{1}{\hat{\sigma}}\left\{\left[\sum_{i=1}^{n}\left(-\frac{x_{i}-\hat{\mu}}{\hat{\sigma}}\right)\right]-n\right\}=0$

$\left.\frac{1}{\hat{\sigma}}\left\{\sum_{i=1}^{n}\left[\left(\frac{x_{i}-\hat{\mu}}{\hat{\sigma}}\right)-\left(\frac{x_{i}-\hat{\mu}}{\hat{\sigma}}\right) \cdot \exp \left(-\frac{x_{i}-\hat{\mu}}{\hat{\sigma}}\right)\right]\right\}-n\right\}=0$

A estimação dos parâmetros da DGVE pode ser obtida utilizando-se uma rotina do software EVIM desenvolvida em MATLAB R11. Referida rotina encontra os parâmetros dessa distribuição pelo método numérico de Newton-Raphson. Podem, entretanto, ocorrer situações de não convergência na estimação de seus parâmetros, sendo que os estimadores de Máxima Verossimilhança - EMV mantêm boas qualidades quando $\xi>-0,5$. ( $\mathrm{SMITH}^{7}$ (1985) apud MENDES (2004, p.46)). Essa afirmação é importante, pois, segundo EMBRECHTS (1999) é comum em aplicações de seguro encontrarem-se valores de $\alpha$ no intervalo $(1 ; 2)-$ que corresponde ao intervalo $(0,5 ; 1)$ para $\xi$ - enquanto em Finanças, $\alpha$ situa-se no intervalo $(2 ; 5)$ - que corresponde ao intervalo $(0,20 ; 0,5)$ para $\xi$.

\subsection{Estimativa de Quantis da DGVE}

Define-se a medida estatística quantil ou p-quantil da variável aleatória $X$, indicada por $Q(p)$, como o valor que satisfaz as relações, $P(X \leq Q(p)) \geq p$ e $P(X \geq Q(p)) \geq 1$ $-p$, em que $0<p<1$.

O p-quantil é, então, o valor tal que a soma das probabilidades dos valores menores do que ele é igual a $p$. A figura $4 \mathbf{O}$, a seguir, representa, graficamente, a estatística p-quantil para uma distribuição contínua.

Considerando que as observações extremas seguem uma DGVE, o p-quantil dessa distribuição é definido como,

$$
\hat{x}_{p}=F_{\hat{\xi}, \hat{\mu}, \hat{\sigma}}^{-1}(p)
$$

Para a equação (1.1) apresentada anteriormente temse que:

$$
\begin{aligned}
& p=\exp \left[-\left(1+\hat{\xi}\left(\frac{\hat{x}_{p}-\hat{\mu}}{\hat{\sigma}}\right)\right)^{-\frac{1}{\hat{\xi}}}\right] \\
& \operatorname{In}(p)=-\left(1+\hat{\xi}\left(\frac{\hat{x}_{p}-\hat{\mu}}{\hat{\sigma}}\right)\right)^{-\frac{1}{\hat{\xi}}} \\
& -\operatorname{In}(p)=\left(1+\hat{\xi}\left(\frac{\hat{x}_{p}-\hat{\mu}}{\hat{\sigma}}\right)\right)^{-\frac{1}{\hat{\xi}}} \\
& \hat{x}_{p}=\hat{\mu}+\frac{\hat{\sigma}}{\hat{\xi}}\left[(-\operatorname{In}(p))^{-\xi}-1\right]
\end{aligned}
$$

\subsection{Valores Extremos Aplicados ao Value-at-Risk (VaR)}

O final da última década caracterizou-se pela ocorrência de significativas crises financeiras no mercado financeiro internacional. Esses acontecimentos demandaram a existência de sistemas de gerenciamento de risco que utilizem metodologias apropriadas para a estimação de eventos raros e com conseqüências financeiras elevadas. Com isso, a TVE experimentou um boom no campo financeiro nos últimos tempos.

As instituições financeiras mantêm um modelo interno de análise de risco de mercado, com destaque especial para o RiskMetrics, desenvolvido pelo Banco americano J.P. Morgan, que teve como resultado final a técnica de mensuração de riscos conhecida como Value-at-Risk (VaR).

O VaR - Valor em Risco — é a perda máxima esperada, em valor monetário, e sob condições normais de mercado, de um título, de uma carteira ou de uma instituição, dado um determinado nível de confiança e um horizonte de tempo especificado.

Segundo Jorion (1998, p. vii), o VaR pode ser descrito como:

VaR é um método de mensuração de risco que utiliza técnicas estatísticas padrões, comumente usadas

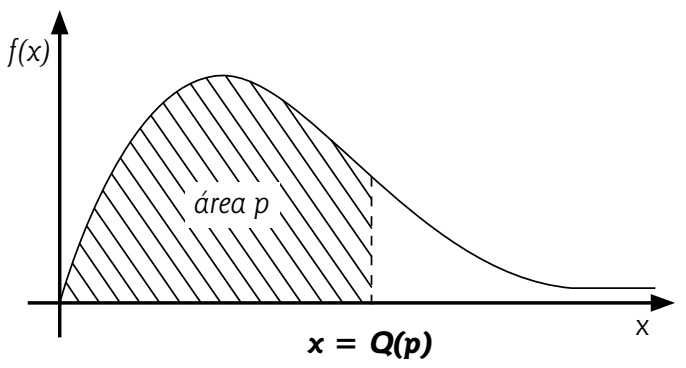

Figura 4

Representação gráfica do p-quantil Q(p) 
em outras áreas técnicas. Em linguagem formal, o VaR mede a pior perda esperada ao longo de determinado intervalo de tempo, sob condições normais de mercado e dentro de determinado nível de confiança. Com base em fundamentos científicos, o VaR fornece aos usuários uma medida concisa do risco de mercado.

\section{Para Morgan (1996, p. 6):}

VaR answers the question: how much can I lose with $x \%$ probability over a given time horizon.

A Teoria de Valores Extremos contribuiu para o aprimoramento dos modelos de VaR por concentrar-se no ajuste da distribuição apenas sobre os valores extremos da variável aleatória, diminuindo, portanto, a influência dos valores centrais. A TVE vem ao encontro da necessidade de se possuir um sistema adequado de controle de risco para prevenir catástrofes financeiras em momentos de crise. Estudos empíricos verificaram o fato de que distribuições de retornos de séries financeiras costumam apresentar caudas mais pesadas do que as de uma distribuição normal. As metodologias tradicionais de cálculo do VaR, contudo, em geral supõem normalidade, seja condicional ou não condicional, e não acomodam, satisfatoriamente, as ocorrências extremas da distribuição dos retornos.

Pelo exposto, o VaR é determinado pelo p-quantil da distribuição dos retornos de um ativo ou de uma carteira de ativos financeiros. Utilizando a TVE para representar a distribuição dos retornos extremos, o VaR Extremo é definido como o $\mathrm{p}$-quantil estimado a partir da distribuição dos valores extremos, DGVE.

De forma particular, o VaR, estimado utilizando-se a Teoria dos Valores Extremos e presumindo-se que a distribuição dos retornos extremos segue uma DGVE, é encontrado a partir da equação (1.7), que determina o p-quantil dessa distribuição. A equação para o VaR extremo é, então,

$$
\operatorname{VaR}_{\text {extremo }}=\hat{\mu}+\frac{\hat{\sigma}}{\hat{\xi}}\left[(-\operatorname{In}(p))^{-\xi}-1\right]
$$

\section{BASE METODOLÓGICA}

As estimativas de perdas máximas pelas diversas metodologias são afetadas pelo erro de estimativa, com isso será verificada a precisão dos modelos com a utilização de um teste de hipótese para a proporção de falhas das estimativas. Referidas falhas serão encontradas comparando-se as perdas futuras estimadas com as perdas efetivamente observadas.

Para as amostras em questão foram desenvolvidos programas em linguagem Fortran e MatlabR12, para o cálculo das estimativas de perdas máximas projetadas utilizandose as seguintes abordagens:

I. Simulação Histórica:

a. construiu-se a distribuição observada dos retornos diários obtidos em 1.000 dias passados;

b. obteve-se o retorno posicionado na cauda esquerda tal que a probabilidade a sua direita corresponda ao nível de confiança desejado.

II. Método Paramétrico com aproximação Normal:

a. calculou-se a média e o desvio-padrão dos retornos diários passados para o tamanho da janela escolhida;

b. obteve-se o valor tabelado da distribuição Normal padrão tal que a área à sua direita corresponda ao nível de confiança desejado;

c. calculou-se o retorno crítico pela seguinte equação:

(média obtida no passo a) + (valor tabelado obtido em b) * (desvio obtido em a)

III. Método Paramétrico utilizando a distribuição logística como representativa para os retornos:

a. estimaram-se os parâmetros da distribuição logística utilizando os retornos passados diários de acordo com o tamanho da janela escolhida, a partir das seguintes equações:

$$
\hat{a}=\bar{x} \quad \hat{b}=\sqrt{\frac{3 \cdot s^{2}}{\pi^{2}}}
$$

em que:

$\bar{x}$ é a média dos retornos para o tamanho da janela móvel escolhida e

$s^{2}$ é a variância dos retornos para o tamanho da janela móvel escolhida.

b. calculou-se a perda máxima esperada pela Logística com os parâmetros estimados em a) para (1 $-\alpha) \%$ de confiança a partir da equação:

$$
\operatorname{VaR}_{\text {logistica }}=\hat{a}+\hat{b} \cdot \operatorname{In}\left(\frac{\alpha}{1-\alpha}\right)
$$

IV. Abordagem TVE utilizando a Distribuição Generalizada dos Valores Extremos - DGVE:

a. determinaram-se os retornos amostrais com base em 1.000 dias úteis;

b. multiplicou-se por (-1) os retornos do passo anterior;

c. estimaram-se os parâmetros da DGVE com a amostra obtida em b) utilizando os máximos obtidos de blocos de 21 dias;

d. estimou-se o VaR extremo, com os parâmetros determinados no passo c, para o nível de confiança $(1-\alpha) \%$ desejado, como segue:

$V a R_{\text {extremo }}=-\left\{\hat{\mu}+\frac{\hat{\sigma}}{\hat{\xi}}\left[(-\operatorname{In}(1-\alpha))^{-\xi}-1\right]\right\}$ 
Empregou-se, adicionalmente, uma janela móvel de 1000 dias úteis para a geração das estimativas de perdas máximas tendo por base os índices Ibovespa, Merval e Dow Jones; a cada dia, a previsão é atualizada agregando-se a informação do primeiro dia à frente e desconsiderando-se a primeira informação da série anterior. $A$ escolha da janela móvel de tamanho fixo igual a 1.000 provém da necessidade de uma amostra de tamanho razoável para a geração de estimativas consistentes dos parâmetros da DGVE, que utiliza apenas os máximos amostrais em sua estimação.

As perdas esperadas calculadas são estimativas influenciadas pela variabilidade amostral; é conveniente, portanto, realizar uma análise de acuidade das metodologias de previsão de perdas, comparando-se a estimativa de perda potencial medida pelas metodologias em pauta com a perda observada após a passagem do tempo. Com isso, para a verificação da precisão das estimativas, conta-se a freqüência com que as perdas potenciais previstas são efetivamente excedidas, comparando-se as perdas projetadas com as realizações observadas; em seguida, utiliza-se um teste de hipóteses para verificar se a freqüência ocorrida encontra-se dentro do intervalo de confiança. Em caso negativo, considera-se que o modelo não é eficaz.
A tabela 1 apresenta o número de estimativas geradas nas simulações e o número de estimativas utilizadas com o propósito de comparação com as perdas efetivamente ocorridas. A intenção da TVE é a previsão de perdas extremas; com isso a verificação dos resultados será realizada em períodos de elevadas perdas. Para as séries financeiras, estipularam-se os meses em que ocorreram as dez piores perdas ocorridas no período de previsão ${ }^{8}$ para a realização do teste.

O método utilizado para verificar a precisão do modelo registra a proporção de falhas, que demonstra a proporção de vezes que a estimativa de perda é excedida em determinada amostra. Segundo Jorion (1997, p.90), Kupiec ${ }^{9}$ (1995) desenvolveu regiões de confiança para esse teste, sendo definidas pelos pontos da cauda da razão de log-verossimilhança:

$$
L R=-2 \operatorname{In}\left[\left(1-p^{*}\right)^{n-x}\left(p^{*}\right)^{x}\right]+2 \operatorname{In}\left[\left(1-\frac{x}{n}\right)^{n-x}\left(\frac{x}{n}\right)^{x}\right]
$$

em que: $p^{*}$ é a probabilidade de perda esperada.

Sob a hipótese nula, a estatística do teste $L R \sim x^{2}{ }_{1 g \mid}$. $A$ hipótese nula significa a adequação do modelo de previsão de perdas.

\section{RESULTADOS}

A tabela 10 expõe os intervalos de confiança do teste de proporção para as séries em pauta. O intervalo $\mathrm{p}$ indica a freqüência de insucessos que poderia ser observada em uma amostra de tamanho n, sem rejeitar a hipótese nula de que $\mathrm{p}^{*}$ é a correta probabilidade a um nível de confiança do teste de $95 \%$. Convém esclarecer que, as perdas esperadas são estimadas com $99 \%$ de confiança (refere-se ao quantil selecionado para a previsão de perdas), logo $p^{*}=1 \%$, enquanto 0 intervalo de não rejeição é construído com $95 \%$ de confiança.

Utilizando-se os intervalos da tabela 1, conclui-se, que para o percentil de $99 \%$ de confiança para previsão das perdas, todos os modelos, com exceção da GVE, estimam incorretamente as perdas em períodos de instabilidade, ao nível de $5 \%$ de significância, conforme atestam as tabelas 2 a 40 .

A tabela 2 revela que, no período em análise, nenhuma perda da série do Ibovespa ultrapassou a previsão de perda máxima estipulada pela Distribuição GVE para o elevado quantil estudado.

Os resultados revelam que todas as abordagens, com exceção da distribuição GVE, subestimam as perdas potenciais considerando períodos de instabilidade e quantis elevados como de 99\% das distribuições de perdas.

\section{1 Resultados para as Três Piores Perdas das Amostras}

Nessa seção, calculam-se as previsões de perdas máximas apenas para os três eventos extremos das séries, ou seja, escolhem-se, inicialmente, as perdas extremas, no caso as três piores perdas, e avalia-se qual seria a estimativa de perda máxima esperada para as alternativas metodológicas. Não se utiliza, nessa seção, a janela fixa de 1000 dados. Como exemplo, tem-se a perda máxima identificada para o Ibovespa, representada pelo retorno de $-17,23 \%$

Tabela 1 Intervalos de Confiança para a Hipótese $\mathrm{H}_{0}$ : $\mathrm{p}^{*}=1 \%$ com $95 \%$ de confiança

\begin{tabular}{|c|c|c|c|}
\hline Descrição & Ibovespa & Merval & Down Jones \\
\hline Tamanho total da amostra & 2446 & 2450 & 2490 \\
\hline Número de previsões simuladas & 1446 & 1450 & 1490 \\
\hline Número de previsões utilizadas para a verificação do modelo & 143 & 183 & 142 \\
\hline Intervalo de confiança & $p<2,80 \%$ & $p<2,73 \%$ & $p<2,82 \%$ \\
\hline
\end{tabular}

Fonte: cálculos dos autores 
Tabela 2 Percentual de ocorrências que excedem o VaR em períodos de alta instabilidade para a Série do Ibovespa

\begin{tabular}{l|c|c} 
Método & $\begin{array}{c}\text { Percentual de Excessos } \\
\text { (quantil 99\% cauda esquerda) }\end{array}$ & $\begin{array}{c}\text { Hipótese } \mathbf{H}_{\mathbf{0}} \text { com 95\% de confiança } \\
\text { (cauda esquerda) }\end{array}$ \\
\hline Simulação Histórica & $3,5 \%$ & Rejeitada \\
\hline Aproximação Normal & $7,7 \%$ & Rejeitada \\
\hline Distribuição Logística & $5,6 \%$ & Rejeitada \\
\hline Distribuição GVE & $0,0 \%$ & Não Rejeitada
\end{tabular}

Fonte: cálculos dos autores

Tabela 3 - Percentual de ocorrências que excedem o VaR em períodos de alta instabilidade para a Série do Merval

\begin{tabular}{l|c|c} 
Método & $\begin{array}{c}\text { Percentual de Excessos } \\
\text { (quantil 99\% cauda esquerda) }\end{array}$ & $\begin{array}{c}\text { Hipótese } \mathbf{H}_{\mathbf{0}} \text { com 99\% de confiança } \\
\text { (cauda esquerda) }\end{array}$ \\
\hline Simulação Histórica & $7,1 \%$ & Rejeitada \\
\hline Aproximação Normal & $8,7 \%$ & Rejeitada \\
\hline Distribuição Logística & $8,7 \%$ & Rejeitada \\
\hline Distribuição GVE & $0,0 \%$ & Não Rejeitada
\end{tabular}

Fonte: cálculos dos autores

Tabela 4 - Percentual de ocorrências que excedem o VaR em períodos de alta instabilidade para a Série do Down Jones

\begin{tabular}{l|c|c} 
Método & $\begin{array}{c}\text { Percentual de Excessos } \\
\text { (quantil 99\% cauda esquerda) }\end{array}$ & $\begin{array}{c}\text { Hipótese } \mathbf{H}_{\mathbf{0}} \text { com 99\% de confiança } \\
\text { (cauda esquerda) }\end{array}$ \\
\hline Simulação Histórica & $7,7 \%$ & Rejeitada \\
\hline Aproximação Normal & $9,2 \%$ & Rejeitada \\
\hline Distribuição Logística & $8,5 \%$ & Rejeitada \\
\hline Distribuição GVE & $0,7 \%$ & Não Rejeitada
\end{tabular}

Fonte: cálculos dos autores

ocorrido em 10/09/1998. Referida perda é o retorno de número 1158 da série Ibovespa, utiliza-se, nesse caso, toda a amostra passada de retornos, 1157 retornos, para o cálculo da estimativa de perda máxima esperada para o dia de número 1158, 10/09/1998.

Apresentam-se, na tabela $5 \bullet$, para as séries estudadas, as três piores perdas ocorridas no período amostral total, bem como as estimativas de perdas máximas esperadas utilizando as diversas metodologias abordadas e considerando alguns níveis de confiança.

Observe-se, na tabela 5, que a primeira perda extrema selecionada está posicionada no sexagésimo dia da série de retornos, portanto, têm-se, apenas, 59 retornos para a estimação da perda máxima esperada. Observa-se na simulação histórica pelo número limitado da amostra que o percentil máximo que pode ser utilizado é de $98 \%$ de confiança (1-1/59), com isso o percentil $99 \%$ está fora da amostra, como identificado.
Observe-se, na tabela 60 , que as datas em que ocorreram as três piores perdas permitiram a inclusão do nível de $99,9 \%$ de confiança na análise. Para o nível de 99,9\% de confiança, observe-se a subestimação em maior grau da previsão perda máxima pelas metodologias de VaR que utilizam as distribuições Normal e Logística em seu bojo. A simulação histórica apresentou para a primeira perda máxima a desvantagem da impossibilidade de estimação.

Pela observação das tabelas 5 a $7 \boldsymbol{\theta}$, conclui-se que as estimativas de perdas máximas esperadas geradas pela distribuição GVE são mais eficazes para as previsões das perdas extremas indicadas pelas outras técnicas.

A grande preocupação com o risco é a capacidade de prever exceções. Dessa forma, as caudas da distribuição de probabilidade são de grande importância do ponto de vista do risco, tornando a TVE de grande valia na estimação mais acurada do risco de perda adicional presente em períodos de alta instabilidade. 
Tabela 5 || Perdas extremas do índice Ibovespa no período amostral total e estimativas de perdas máximas esperadas considerando diversos níveis de confiança

\begin{tabular}{|c|c|c|c|c|}
\hline \multicolumn{2}{|l|}{ Retornos } & dia da ocorrência & \multicolumn{2}{|c|}{ data da ocorrência } \\
\hline \multicolumn{2}{|l|}{$-11,19 \%$} & 60 & \multicolumn{2}{|c|}{ 04/04/1994 } \\
\hline \multicolumn{2}{|l|}{$-16,22 \%$} & 943 & \multicolumn{2}{|c|}{ 27/10/1997 } \\
\hline \multicolumn{2}{|l|}{$-17,23 \%$} & 1158 & \multicolumn{2}{|c|}{ 10/09/1998 } \\
\hline \multicolumn{5}{|c|}{$1^{\text {a }}$ perda extrema, $-11,19 \%$, correspondente ao retorno $n^{\circ} 60$ da amostra } \\
\hline Nível de Confiança & SH & Normal & Logística & GVE \\
\hline $95,0 \%$ & $-5,02 \%$ & $-4,71 \%$ & $-4,62 \%$ & $-8,51 \%$ \\
\hline $97,5 \%$ & $-8,09 \%$ & $-6,07 \%$ & $-6,33 \%$ & $-10,63 \%$ \\
\hline $99,0 \%$ & fora da amostra & $-7,64 \%$ & $-8,54 \%$ & $-13,57 \%$ \\
\hline \multicolumn{5}{|c|}{$2^{a}$ perda extrema, $-16,22 \%$, correspondente ao retorno $n^{\circ} 943$ da amostra } \\
\hline Nível de Confiança & SH & Normal & Logística & GVE \\
\hline $95,0 \%$ & $-4,41 \%$ & $-4,63 \%$ & $-4,56 \%$ & $-11,90 \%$ \\
\hline $97,5 \%$ & $-5,52 \%$ & $-5,58 \%$ & $-5,77 \%$ & $-16,41 \%$ \\
\hline $99,0 \%$ & $-8,73 \%$ & $-6,70 \%$ & $-7,33 \%$ & $-24,92 \%$ \\
\hline \multicolumn{5}{|c|}{$3^{\mathrm{a}}$ perda extrema, $-17,23 \%$, correspondente ao retorno $n^{\circ} 1158$ da amostra } \\
\hline Nível de Confiança & SH & Normal & Logística & GVE \\
\hline $95,0 \%$ & $-4,69 \%$ & $-4,83 \%$ & $-4,77 \%$ & $-12,40 \%$ \\
\hline $97,5 \%$ & $-6,06 \%$ & $-5,80 \%$ & $-5,99 \%$ & $-16,49 \%$ \\
\hline $99,0 \%$ & $-9,08 \%$ & $-6,93 \%$ & $-7,57 \%$ & $-23,68 \%$ \\
\hline
\end{tabular}

Fonte: cálculos dos autores

Tabela 6 | Perdas extremas do índice Merval no período amostral total e estimativas de perdas máximas esperadas considerando diversos níveis de confiança

\begin{tabular}{|c|c|c|c|c|}
\hline \multicolumn{2}{|l|}{ Retornos } & \multicolumn{2}{|l|}{ dia da ocorrência } & data da ocorrência \\
\hline \multicolumn{2}{|l|}{$-14,77 \%$} & 959 & \multicolumn{2}{|c|}{ 27/10/1997 } \\
\hline \multicolumn{2}{|l|}{$-14,30 \%$} & 1175 & \multicolumn{2}{|c|}{$10 / 09 / 1998$} \\
\hline \multicolumn{2}{|l|}{$-13,20 \%$} & 1976 & \multicolumn{2}{|c|}{$28 / 11 / 2001$} \\
\hline \multicolumn{5}{|c|}{$1^{\text {a }}$ perda extrema, $-14,77 \%$, correspondente ao retorno $n^{\circ} 959$ da amostra } \\
\hline Nível de Confiança & SH & Normal & Logística & GVE \\
\hline $95,0 \%$ & $-3,50 \%$ & $-3,45 \%$ & $-3,40 \%$ & $-7,76 \%$ \\
\hline $97,5 \%$ & $-4,93 \%$ & $-4,11 \%$ & $-4,24 \%$ & $-8,93 \%$ \\
\hline $99,0 \%$ & $-6,01 \%$ & $-4,89 \%$ & $-5,33 \%$ & $-10,49 \%$ \\
\hline $99,9 \%$ & fora & $-6,51 \%$ & $-8,02 \%$ & $-14,48 \%$ \\
\hline \multicolumn{5}{|c|}{$2^{a}$ perda extrema, $-14,30 \%$, correspondente ao retorno $n^{\circ} 1175$ da amostra } \\
\hline Nível de Confiança & SH & Normal & Logística & GVE \\
\hline $95,0 \%$ & $-3,98 \%$ & $-3,75 \%$ & $-3,70 \%$ & $-9,06 \%$ \\
\hline $97,5 \%$ & $-5,01 \%$ & $-4,46 \%$ & $-4,59 \%$ & $-10,78 \%$ \\
\hline $99,0 \%$ & $-6,35 \%$ & $-5,28 \%$ & $-5,75 \%$ & $-13,24 \%$ \\
\hline $99,9 \%$ & $-14,15 \%$ & $-7,01 \%$ & $-8,62 \%$ & $-20,61 \%$ \\
\hline \multicolumn{5}{|c|}{$3^{a}$ perda extrema, $-13,20 \%$, correspondente ao retorno $n^{\circ} 1976$ da amostra } \\
\hline Nível de Confiança & SH & Normal & Logística & GVE \\
\hline $95,0 \%$ & $-4,03 \%$ & $-3,89 \%$ & $-3,84 \%$ & $-9,48 \%$ \\
\hline $97,5 \%$ & $-5,19 \%$ & $-4,62 \%$ & $-4,76 \%$ & $-11,41 \%$ \\
\hline $99,0 \%$ & $-6,51 \%$ & $-5,48 \%$ & $-5,96 \%$ & $-14,25 \%$ \\
\hline $99,9 \%$ & $-14,31 \%$ & $-7,26 \%$ & $-8,93 \%$ & $-23,19 \%$ \\
\hline
\end{tabular}


Tabela 7 - Perdas extremas do índice Down Jones no período amostral total e estimativas de perdas máximas esperadas considerando diversos níveis de confiança

\begin{tabular}{|c|c|c|c|c|}
\hline \multicolumn{2}{|l|}{ Retornos } & dia da ocorrência & \multicolumn{2}{|c|}{ data da ocorrência } \\
\hline \multicolumn{2}{|l|}{$-7,45 \%$} & 964 & \multicolumn{2}{|c|}{ 27/10/1997 } \\
\hline \multicolumn{2}{|l|}{$-6,58 \%$} & 1176 & \multicolumn{2}{|c|}{$31 / 08 / 1998$} \\
\hline \multicolumn{2}{|c|}{\begin{tabular}{l|l}
$-7,40 \%$ &
\end{tabular}} & 1940 & \multicolumn{2}{|c|}{$17 / 09 / 2001$} \\
\hline \multicolumn{5}{|c|}{$1^{\text {a }}$ perda extrema, $-7,45 \%$, correspondente ao retorno $n^{\circ} 964$ da amostra } \\
\hline Nível de Confiança & SH & Normal & Logística & GVE \\
\hline $95,00 \%$ & $-1,21 \%$ & $-1,18 \%$ & $-1,16 \%$ & $-2,89 \%$ \\
\hline $97,50 \%$ & $-1,69 \%$ & $-1,42 \%$ & $-1,47 \%$ & $-3,34 \%$ \\
\hline $99,00 \%$ & $-2,30 \%$ & $-1,70 \%$ & $-1,86 \%$ & $-3,95 \%$ \\
\hline $99,90 \%$ & fora & $-2,28 \%$ & $-2,83 \%$ & $-5,52 \%$ \\
\hline $99,99 \%$ & fora & $-2,76 \%$ & $-3,80 \%$ & $-7,21 \%$ \\
\hline \multicolumn{5}{|c|}{$2^{a}$ perda extrema, $-6,58 \%$, correspondente ao retorno $n^{\circ} 1176$ da amostra } \\
\hline Nível de Confiança & SH & Normal & Logística & GVE \\
\hline $95,00 \%$ & $-1,31 \%$ & $-1,34 \%$ & $-1,32 \%$ & $-3,66 \%$ \\
\hline $97,50 \%$ & $-1,71 \%$ & $-1,61 \%$ & $-1,66 \%$ & $-4,52 \%$ \\
\hline $99,00 \%$ & $-2,36 \%$ & $-1,93 \%$ & $-2,10 \%$ & $-5,85 \%$ \\
\hline $99,90 \%$ & $-6,90 \%$ & $-2,58 \%$ & $-3,19 \%$ & $-10,53 \%$ \\
\hline \multicolumn{5}{|c|}{$3^{\mathrm{a}}$ perda extrema, $-7,40 \%$, correspondente ao retorno $n^{\circ} 1940$ da amostra } \\
\hline Nível de Confiança & SH & Normal & Logística & GVE \\
\hline $95,00 \%$ & $-1,64 \%$ & $-1,64 \%$ & $-1,62 \%$ & $-4,12 \%$ \\
\hline $97,50 \%$ & $-2,17 \%$ & $-1,97 \%$ & $-2,03 \%$ & $-4,94 \%$ \\
\hline $99,00 \%$ & $-2,71 \%$ & $-2,34 \%$ & $-2,56 \%$ & $-6,13 \%$ \\
\hline $99,50 \%$ & $-6,63 \%$ & $-2,60 \%$ & $-2,95 \%$ & $-7,12 \%$ \\
\hline
\end{tabular}

Fonte: cálculos dos autores

\section{CONCLUSÕES}

Investigou-se, neste trabalho, a estimação de perdas máximas esperadas para séries financeiras tendo por base:

- as metodologias tradicionais, que utilizam todos os dados amostrais na estimação da variável aleatória em questão e

- a metodologia dos Valores Extremos, que utiliza, apenas, os máximos amostrais na estimação da previsão de perdas máximas esperadas.

Para tais séries, constatou-se que:

- o VaR Normal subestima a exposição ao risco de mercado para níveis de confiança elevados;

- a hipótese de normalidade não é adequada para representar ao retornos das amostras analisadas;

- a distribuição Logística apresentou o melhor ajuste em detrimento de outras distribuições contidas no Software Bestfit, seguindo os testes K-S e A-D, entretanto, todas as distribuições ajustadas, utilizando-se a amostra total de dados, foram rejeitadas. O ajuste se torna mais inadequado à medida que se aproxima das caudas;

- a utilização da distribuição empírica, simulação histórica, construída a partir da amostra de dados, apresenta, como desvantagem, dependendo do tamanho da amostra $n$, a impossibilidade de estimação de quantis inferiores a $1 / n$;
- a hipótese do ajuste pela distribuição GVE aos retornos mínimos amostrais não foi rejeitada, ao nível de significância de 5\%;

- a aplicação da metodologia TVE mostrou-se satisfatória para a estimação das perdas esperadas em períodos de instabilidade financeira considerando elevados níveis de confiança.

Ressalte-se que o VaR, obtido pelos diversos métodos apresentados, é apenas uma estimativa do verdadeiro valor em risco, que é obviamente desconhecido. Deve-se estar ciente da presença do erro de estimativa no cálculo do VaR. A estimação de um valor para o VaR não deve constituir, portanto, o único referencial para o controle e gestão dos riscos de mercado.

É mister observar se há semelhança entre o período histórico utilizado para o cálculo do VaR e o período futuro para o qual se está estimando a volatilidade, considerando-se que a volatilidade dos retornos de um ativo se modifica ao longo do tempo.

O objetivo de um sistema de gestão de risco consiste em determinar o montante de capital que um investidor deverá manter de forma a garantir o cumprimento de uma obrigação financeira. Essa decisão envolve um trade-off: 1) se o montante do capital a ser resguardado for fixado em 
um nível demasiadamente elevado, os investidores não serão incentivados a entrar no mercado; 2) se o montante de capital for fixado a um nível demasiadamente baixo, existe o risco de o investidor não cumprir suas obrigações em condições adversas. Com isso, torna-se extremamente importante a escolha da correta metodologia de VaR em conjunto com o nível de confiança desejado.

Sugere-se para estudos posteriores a utilização da Volatilidade Condicional em conjunto com a Teoria dos Valores Extremos para acomodar a massa de probabilidade adicional presente em períodos de alta volatilidade.

Em períodos de estabilidade financeira, em condições normais de mercado, as metodologias convencionais de estimação do VaR são satisfatórias. Com isso, Coronado (2000, p. 3) revela que a Teoria dos Valores Extremos é de grande relevância para se somar aos métodos existentes de mensuração do VaR:
En conclusión, la Teoría de los Valores Extremos sirve como análisis complementario del VaR y no como "sustituto" del VaR. Este es, también, el espíritu de la frase que el presidente de la Reserva Federal, Alan Greenspan, pronuncia en la Joint Central Bank Conference "Risk Measurement and Systemic Risk", del Board of Governors of the Federal Reserve System, el 16 de noviembre de 1996 en Washington D.C.:

"Work that characterizes the statistical distribution of extreme events would be useful, as well".

Finalmente, a base teórica utilizada mostrou-se eficaz em sanar a necessidade premente de quantificar de forma adequada reservas para suportar perdas extremas no mercado financeiro.

\section{Referências Bibliográficas}

BAUTISTA, E. A. L.. A distribuição Generalizada de Valores Extremos no estudo da velocidade máxima do vento em Piracicaba. 2002. Dissertação de Mestrado em Agronomia. Universidade de São Paulo, Piracicaba, São Paulo.

CHAVEZ, V.; ROEHRL, A.. Extreme Value Theory can save your neck, 2004. Disponível em $<$ http://www.approximity.com/papers/TVE wp.pdf >. Acesso em: 25 de janeiro de 2005.

CORONADO, María. Extreme Value Theory (TVE) for Risk Managers: Pitfalls and Opportunities. In: The Use of TVE in Measuring VaR, Madrid: Facultad de Ciencias Económicas y Empresariales. Universidad P. Comillas de Madrid, 2000.

EMBRECHTS, P. et al.. Extreme Value Theory as a Risk Management Tool. 1998. ETH preprint. Disponível em:<www.math.ethz.ch/ embrechts>. Acesso em: 18 de dezembro de 2003.

JORION, Philippe. Value at Risk: A Nova Fonte de Referência para o Controle do Risco de Mercado. São Paulo: Bolsa de Mercadorias \& Futuros, 1998

J.P. Morgan Bank. Riskmetrics Technical Manual. 4 ed., 1996. J. P. Morgan Bank: New York. Disponível em:<http://www.riskmetrics.com/ research/techdocs $>$. Acesso em: 11 de outubro de 2003.

KUPIEC, P.. Techniques for Verifying the Accuracy of Risk Measurement Models. Journal of Derivatives 2, p. 73-84, dez. 1995.

MENDES, Beatriz V. de M.. Uma Introdução à Analise de Valores Extremos. Rio de Janeiro: E-papers Serviços Editoriais, 2004

SMITH, R. L.. Maximum Likelihood Etimation in a class of nonregular cases. Biometrika, n. 72, p. 67-90. 1985.

SOUZA, Luiz Alvarez Rezende. Valor em Risco em Épocas de Crise. 1999. (Dissertação de Mestrado em Economia). Faculdade de Economia, Administração e Contabilidade. Universidade de São Paulo, São Paulo.

\section{NOTA - Endereço dos autores}

Universidade Federal do Ceará

Av. da Universidade, 2700 - $2^{\circ}$ andar

Forteleza - CE

60.020-181 